\title{
A meteo-hydrological forecasting chain: performance of the downscaling and rainfall-runoff steps in a small catchment
}

\author{
R. Deidda, M. G. Badas, A. Seoni, and E. Piga \\ Dipartimento di Ingegneria del Territorio, Università di Cagliari, Piazza d'Armi, 09123 Cagliari, Italy \\ Received: 11 November 2005 - Revised: 6 June 2006 - Accepted: 19 July 2006 - Published: 19 September 2006
}

\begin{abstract}
Forecasting ground effects of severe meteorological events with an adequate lead time is fundamental for civil protection scopes and is therefore an important challenge for the scientific community. The paper focuses on the performance of some steps of a meteo-hydrological forecasting chain that can be applied in small watersheds to assess hydrological risk deriving by an intense storm predicted at the large meteorological scale. The proposed procedure integrates large-scale rainfall fields, as those produced by numerical weather prediction (NWP) models, with statistical rainfall downscaling and hydrological modelling. More in details, assuming a large scale rain rate as the input of the process, the forecasting chain produces an ensemble of hydrographs that are post-processed in order to give a probabilistic representation of mean streamflow maxima for different time windows. The outcome of this procedure can be thus applied to assess the risk that some critical streamflow thresholds may be exceeded. The procedure has been tested on more than one thousand recorded events in the Araxisi catchment in Sardinia, Italy. Results and performances are presented and discussed.
\end{abstract}

\section{Introduction}

In order to properly identify and manage hydrogeological risk, potential ground effects caused by intense meteorological events have to be predicted with an adequate lead time. In case of small watersheds this aim cannot be achieved by means of rainfall and streamflow real time telemetered observations. In fact, short catchment response times do not allow monitoring systems to provide flood forecast in time to put on the alert and to set up the required protection measures. This task can be pursued by means of forecasting chains that integrate Quantitative Precipitation Forecast (QPF) given by $\mathrm{Nu}-$ merical Weather Prediction (NWP) models and hydrological

Correspondence to: R. Deidda

(rdeidda@unica.it) modelling. The scientific community is therefore focusing its attention on this issue in order to develop effective procedures for civil protection (Ferraris et al. (2002) and Siccardi et al. (2005) among the others).

A forecasting chain designed to evaluate, in a probabilistic framework, the hydrological risks connected to a predicted meteorological event is depicted in Fig. 1: the oval shapes represent the procedure steps while the rectangles show the information passing from one step to the next one. Actually, information flows through different kinds of models: NWP model (step 1), downscaling model (step 2), rainfall-runoff model (step 3) and postprocessing model (step 4). The forecasting chain can be viewed as a Monte Carlo system that allows determining the exceedance probability associated to a given flow peak threshold or to a given discharge volume flowing in a fixed time window.

Rainfall fields predicted by NWP models at step 1 represent the initial information useful for the hydrological steps of the forecasting chain. Nevertheless meteorological and hydrological models should not be directly coupled to forecast floods in small watersheds. Actually, due to numerical diffusion, NWP models are not able to provide reliable predictions at their own grid resolution, thus rainfall fields need to be reaggregated to a coarser resolution. The gap between these large scales where NWP predictions become reliable and the small scales required by the hydrological modelling should be filled in by downscaling procedures (step 2 in Fig. 1). Multifractal models can be successfully applied at this scope, since they are able to reproduce rainfall probability distributions at unresolved small scales. Starting from a precipitation event predicted over a large space-time scale by a NWP model, multifractal models allow generating a set of several equally probable rainfall realizations at scales compatible with rainfall-runoff modelling. Uncertainty due to unresolved space and time small scales is thus described with a statistical approach in a Monte Carlo system.

At step 3 each (synthetically downscaled) rainfall scenario is transformed into a corresponding hydrograph at a catchment outlet by means of a rainfall-runoff model. In such a 


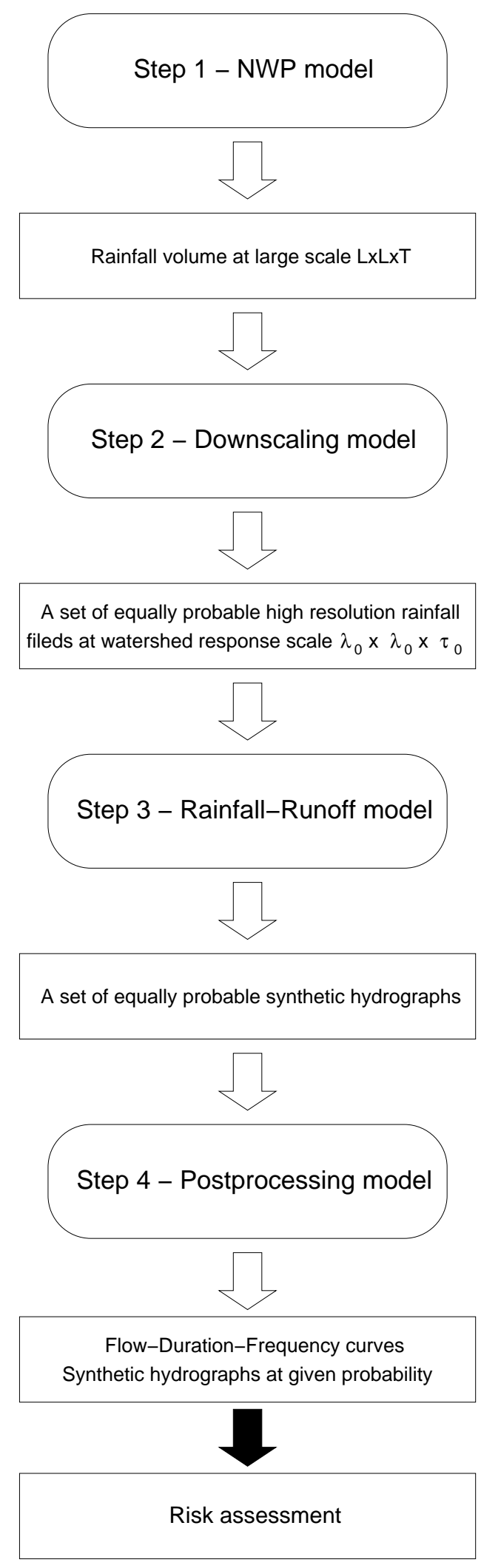

Fig. 1. The proposed forecasting chain structure is illustrated: the oval shapes represent the procedure steps while the rectangles show the information passing from a step to the next one. way, a set of equally probable hydrographs is generated starting from the same meteorological prediction.

In order to properly organize civil protection operations, one would know whether or not a given threshold for flow peak or discharge volume will be exceeded. Unfortunately, due to uncertainties within the forecasting process (related to unresolved scales or to the models characteristics), the answer cannot simply be yes or not, but it can be given only in a probabilistic framework. This issue is tackled in step 4 with the postprocessing model, which allows also determining the exceedance probability of discharge volumes flowing in a range of different time windows.

Steps 2, 3 and 4 of the forecasting chain are evaluated for a case study, the Araxisi catchment. We deliberately jump the step 1 in order to avoid the propagation of errors and uncertainties related to the meteorological forecast, and to better assess the performances of the downscaling and hydrological steps. Anyhow, it should be clear that the proposed forecasting chain can be operatively applied starting from NWP forecasted rainfall fields. The downscaling model (step 2) is briefly illustrated, applied and tested in Sect. 2; the hydrological models performance (step 3) is analyzed in Sect. 3; while Sect. 4 is devoted to the postprocessing model (step 4). Finally in Sect. 5 the conclusions of the work are drawn.

\section{Downscaling model (step 2)}

As discussed above, NWP models alone are unlikely to provide rainfall fields with an adequate and reliable resolution for an efficient application of rainfall-runoff models to small basins. Multifractal theory has been proved to be a suitable framework for rainfall downscaling as witnessed by many works in literature (Lovejoy and Mandelbrot (1985); Schertzer and Lovejoy (1987); Gupta and Waymire (1993); Over and Gupta (1996); Perica and Foufoula-Georgiou (1996); Deidda (2000) among the others). A multifractal model, able to correctly reproduce the observed small scale rainfall variability, was therefore chosen as the second step of the proposed forecasting chain. In the following, after some brief recalls to multifractal theory, we present the application and validation of the downscaling step on the Araxisi catchment.

\subsection{Downscaling model - theory}

Let $L, \lambda_{0}<L$ be linear spatial scales and $T, \tau_{0}<T$ be times scales. Given the rainfall volume (or mean rain rate $I$ ) over the large scales $L \times L \times T$ resolved by meteorological models (it may be also a re-aggregated output coming from high resolution NWP model), a multifractal downscaling model should be able to reproduce the probability distribution function of rainfall volumes over smaller scales $\lambda_{0} \times \lambda_{0} \times \tau_{0}$ compatible with the watershed space-time response scales.

Let $i(x, y, t)$ be the rainfall intensity at location $(x, y)$ and time $t$. Scale invariance properties are investigated on the rainfall volumes $\mu$ defined within a generic space-time 
region $\lambda_{x} \times \lambda_{y} \times \tau$ located in $x_{i}, y_{j}, t_{k}$ (and smaller than the large scale $L \times L \times T$ ), which can be written as:

$\mu_{i, j, k}\left(\lambda_{x}, \lambda_{y}, \tau\right)=\int_{x_{i}}^{x_{i}+\lambda_{x}} d x \int_{y_{j}}^{y_{j}+\lambda_{y}} d y \int_{t_{k}}^{t_{k}+\tau} d t i(x, y, t)$

Usually, spatial isotropy is assumed, letting the two spatial scales equal to $\lambda: \lambda_{x}=\lambda_{y}=\lambda$. Also the integration time scale $\tau$ in Eq. (1) can be related to the spatial scale $\lambda$ by the relationship $\tau=\lambda / U$, where $U$ is a space-time conversion parameter that may be or not a function of $\lambda$. In the simplest scenario of self-similarity (or scale isotropy) $U$ is a scaleindependent parameter that can be introduced in order to transfer the statistical properties observed at space scales $\lambda$ to coherent time scales $\tau=\lambda / U$ and vice versa. In this case the measure defined in Eq. (1) becomes a function of $\lambda$ scale only: $\mu_{i, j, k}\left(\lambda_{x}, \lambda_{y}, \tau\right)=\mu_{i, j, k}(\lambda)$. Hence the following $q$ order partition functions $S_{q}(\lambda)$ can be computed at different scales $\lambda$ :

$S_{q}(\lambda)=\frac{1}{N(\lambda)^{2} N(\tau)} \sum_{i=1}^{N(\lambda)} \sum_{j=1}^{N(\lambda)} \sum_{k=1}^{N(\tau)} \mu_{i, j, k}(\lambda)^{q}$

where $N(\lambda)^{2} N(\tau)$ is the number of subregions $\lambda \times \lambda \times \tau$ of the chosen $\lambda$-partition, being $N(\lambda)$ and $N(\tau)=N(\lambda)$ the number of subgrid cells in each direction of space and time respectively.

A linear trend of partition functions $S_{q}(\lambda)$ versus $\lambda$ in a log-log plane identifies the existence of a scale-invariance range where $S_{q}(\lambda) \sim \lambda^{\zeta(q)}$. Moreover, if multifractal exponents $\zeta(q)$ are non linear functions of $q$, the measure $\mu$ is multifractal. This kind of behaviour was detected on spacetime rainfall events retrieved by radar or by high density raingauges network (see Deidda (2000); Deidda et al. (2004); Badas et al. (2005, 2006) among the others). The identification of scale-invariant properties allows the calibration of multifractal models, whose parameters are usually estimated fitting sample multifractal moments $\zeta(q)$ to their theoretical behaviour, which can be derived from the model generator structure.

The STRAIN model (Deidda et al., 1999; Deidda, 2000) was here chosen to reproduce the analysed rain fields. The model was successfully applied in its simplest form for the generation of homogeneous space-time rain fields in sites where orography does not exert any significant conditioning on rainfall patterns (Deidda, 2000; Deidda et al., 2004, 2005). Badas et al. (2005) recently analysed several precipitation events occurred over complex terrain in the Sardinia region and showed that the observed spatial heterogeneity due to the orography can be reproduced through the superimposition of a modulating function $\bar{\xi}$ on generated fields:

$i(x, y, t)=\bar{\xi}(x, y) i_{0}(x, y, t)$

where $i_{0}(x, y, t)$ represents the homogeneous and isotropic rain rate field generated by the STRAIN model at location $(x, y)$ and time $t$, the dimensionless modulating function $\bar{\xi}(x, y)$ is computed on the basis of the corresponding terrain elevation $z(x, y)$, and $i(x, y, t)$ is the resulting rain intensity that takes into account orography conditioning.

\subsection{Downscaling model - application}

Although over the last decade the skill of NWP systems to reproduce physical processes has increased significantly (Simmons and Hollingsworth, 2002), precipitation is still one of the most difficult atmospheric parameters to forecast. Therefore, in order to better assess performances and uncertainties of the downscaling and hydrological steps, in the study case we used observed rainfall data instead of NWP forecast, thus jumping the step 1 in the application of the proposed procedure, as anticipated in the Introduction. In such a way, performances of step 2 and 3 are not affected by NWP errors.

The rainfall database used here consists of daily records observed in 394 stations distributed all over Sardinia in the period (1982-1980). Thus the large scales of the downscaling process are $T=24 \mathrm{hr}$ in time (daily data) and consequently $L=U T=416 \mathrm{~km}$ in space, having assumed a constant space-time homogenization parameter $U=17.33 \mathrm{~km} / \mathrm{hr}$ (Badas et al., 2005). Mean rainfall intensities $I$ on this large space-time domain $L \times L \times T$ were computed for each day averaging all daily data of the rain gage network of the Sardinian Hydrological Survey. The most intense rainfall events were selected on the basis of a threshold mean intensity $I=5 \mathrm{~mm} /$ day. The downscaling process was then applied starting from the large scale rain rate $I$ of those events whose corresponding hydrograph records at the Araxisi catchment outlet were also available. 1303 events were found to satisfy both criteria on rainfall threshold and stramflow measures availability. The downscaling procedure was thus applied on these events from scales $L=416 \mathrm{~km}$ and $T=24 \mathrm{hr}$ up to scales $\lambda_{0}=13 \mathrm{~km}$ and $\tau_{0}=45 \mathrm{~min}$, being $\lambda_{0}^{2}$ close to the Araxisi area $\left(125 \mathrm{~km}^{2}\right)$.

For each of the selected events, the STRAIN model was applied 3000 times in order to numerically reproduce the variability (and more in general the statistical properties) of rainfall fields up to $\lambda_{0}$ and $\tau_{0}$ scales. In such a way, 3000 synthetic samples, each one containing 1303 downscaled events, were obtained. The STRAIN model is based on a logPoisson generator $\mu=\beta^{y}$, where $y$ is a Poisson distributed i.i.d. random variable with mean $c$. Multifractal analyses on several datasets (Deidda (2000); Deidda et al. (2004, 2005); Badas et al. (2005)) have shown that the $\beta$ parameter can be assumed equal to $\exp (-1)$, becoming a model constant, while sample estimates of the $c$ parameter follow decreasing functions of the large scale rainfall rate $I$. For the Sardinia region a $c(I)$ relationship was determined by Badas et al. (2005): it is used here with a slight modification in the coefficients due to the larger domain $L \times L \times T$. Homogeneous and isotropic rainfall fields $i_{0}(x, y, t)$ generated by the STRAIN model up to scales $\lambda_{0}$ and $\tau_{0}$ are then transformed into orographic conditioned fields $i(x, y, t)$ by equation Eq. (3). The required modulating function $\bar{\xi}(x, y)$ was estimated on the basis of each grid cell mean elevation following Badas et al. (2005). The value $\bar{\xi}=1.35$ resulted for the elevation of the grid cell $\lambda_{0} \times \lambda_{0}(13 \mathrm{~km} \times 13 \mathrm{~km})$ corresponding to the Araxisi catchment. 
Table 1. Intensity ranges and numerousness of the classes used in the validation process.

\begin{tabular}{ccccccc}
\hline class & 1 & 2 & 3 & 4 & 5 & 6 \\
I [mm/day] & $5-7$ & $7-10$ & $10-15$ & $15-20$ & $20-30$ & $>30$ \\
\# of events & 385 & 335 & 310 & 136 & 97 & 40 \\
\hline
\end{tabular}

From each downscaled space-time field, the time history (rainfall hyetograph) over the Araxisi grid cell $\left(\lambda_{0} \times \lambda_{0}\right)$ was selected: 3000 synthetic hyetographs were thus extracted for each of the alalysed events. As an example, in Fig. 2 two generated hyetographs (having resolution $\tau_{0}=45 \mathrm{~min}$ ) are shown as possible realization of the same large scale event. Fig. 2 clearly shows how downscaled hyetographs of the same large scale event may differ not only for the inner sub-daily variability, but also for daily total depth (due to simultaneous downscaling in space and time).

\subsection{Downscaling model - validation}

The performance of each step of the proposed forecasting procedure has been tested in order to identify possible critical points. With the aim to perform a comparison between modelled and observed data, the 1303 selected events were grouped into six classes on the basis of large scale mean intensity $I$. Intensity ranges and numerousness of each class are reported in Table 1. This subdivision, which has been used also in Sect. 3, allows grouping similar events in terms of STRAIN model parameters, since $c$ is related to large scale mean intensity $I$. Performance evaluation of each step of the flood forecasting procedure has been pursued comparing the cumulative distribution function (CDF) obtained for observed and synthetic data within each class.

The absence of sub-daily observed rainfall data imposed to carry on the comparison on a daily scale. The control dataset of precipitation occurring over the catchment during the selected events was computed from daily rain gage data by means of the Thiessen method. On the other side, downscaled rainfall intensities generated on a spatial scale $\lambda_{0}^{2}$ close to the catchment area and with time resolution $\tau_{0}=45 \mathrm{~min}$, were integrated at the same daily scale as the control set.

The comparison between observed and synthetic data was performed as follows. Let $N$ be the number of analysed events in each class reported in Table 1 . We have on one hand a sample of $N$ observed values (e.g. for class 1 we have 385 values of areal average daily rainfall depth), and on the other hand 3000 synthetic samples each one containing $N$ generated values (i.e. rainfall downscaled at $\lambda_{0} \times \lambda_{0}$ scale and reaggregated at the daily scale). Data of each sample (the one observed and the 3000 simulated) are sorted in ascending order $\left(x_{1}<x_{2}<\cdots<x_{N}\right)$ becoming an order statistics series. Our purpose is to estimate an equitailed confidence interval of the $r$-th order statistics $(r=1 \cdots N)$. Confidence limits are thus obtained from the quantiles $q_{a}$ and $q_{1-a}$ of the empirical distribution of the corresponding $3000 r$-th order statistics in synthetic samples.
As an example, Fig. 3 illustrates the comparison for the third class: empirical CDF of the control dataset (daily precipitation values observed on the Araxisi catchment) is compared with the $90 \%$ confidence limits estimated from generated sequences (i.e. CDFs of quantiles $q_{0.05}$ and $q_{0.95}$ of the $r$-th order statistics). The logarithmic scale was chosen in order to emphasize intense precipitation values. In the left plot quantiles refer to spatially homogeneous rainfall fields generated by the STRAIN model, while the modulating function $\bar{\xi}$ is included in data plotted in the right image. It is now apparent how the introduction of the modulating function is essential for a correct reproduction of observed data in domains with complex orography. Actually, order statistics of observed data are within the confidence limits derived from synthetic data including $\bar{\xi}$ value, as it is apparent in Fig. 3 for the third class. Similar results were obtained for all the other analysed classes, thus synthetic hyetographs including the modulating function properly represent observed events at least at a daily scale and were used in the next step of the forecasting chain.

\section{Rainfall-runoff model (step 3)}

\section{$3.1 \quad$ R-R model - theory}

In the third step of the forecasting chain, hyetographs generated by the downscaling model need to be transformed into hydrographs at the catchment outlet. This rainfallrunoff transformation involves many hydrological processes. Among the large number of rainfall-runoff models describing with different levels of approximation the physical aspects of these processes, we choose here a very simple and widely used approach. It consists first in the application of SCS curve number (SCS-CN) method (SCS, 1972) to derive excess rainfall from observed rainfall, and then in the use of the unit hydrograph (UH) theory to represent the transformation of excess rainfall hyetograph into the corresponding direct runoff hydrograph.

The SCS method is based on the well known equation:

$$
P_{e}=\frac{\left(P-I_{a}\right)^{2}}{\left(P-I_{a}\right)-S}
$$

where $P_{e}$ is the excess rainfall, $P$ is the observed rainfall, $S$ is the potential maximum retention after runoff begins and $I_{a}$ is the initial abstraction. Usually the initial abstraction is assumed as a fraction of $S\left(I_{a}=0.2 S\right)$, in this case $S$ is the only parameter that needs to be determined in the SCS$\mathrm{CN}$ equation. The potential maximum retention $S$ is related to a dimensionless $\mathrm{CN}$ parameter, ranging between 0 and 100: in case all the variables of Eq. (4) are expressed in $\mathrm{mm}$, this relationship becomes $S=(25400 / C N)-254$. Once the $\mathrm{CN}$ value is determined on the basis of hydrological soil groups, land-cover and antecedent moisture condition (AMC), Eq. (4) allows to determine the excess rainfall hyetograph corresponding to each total rainfall hyetograph coming from the downscaling model. 

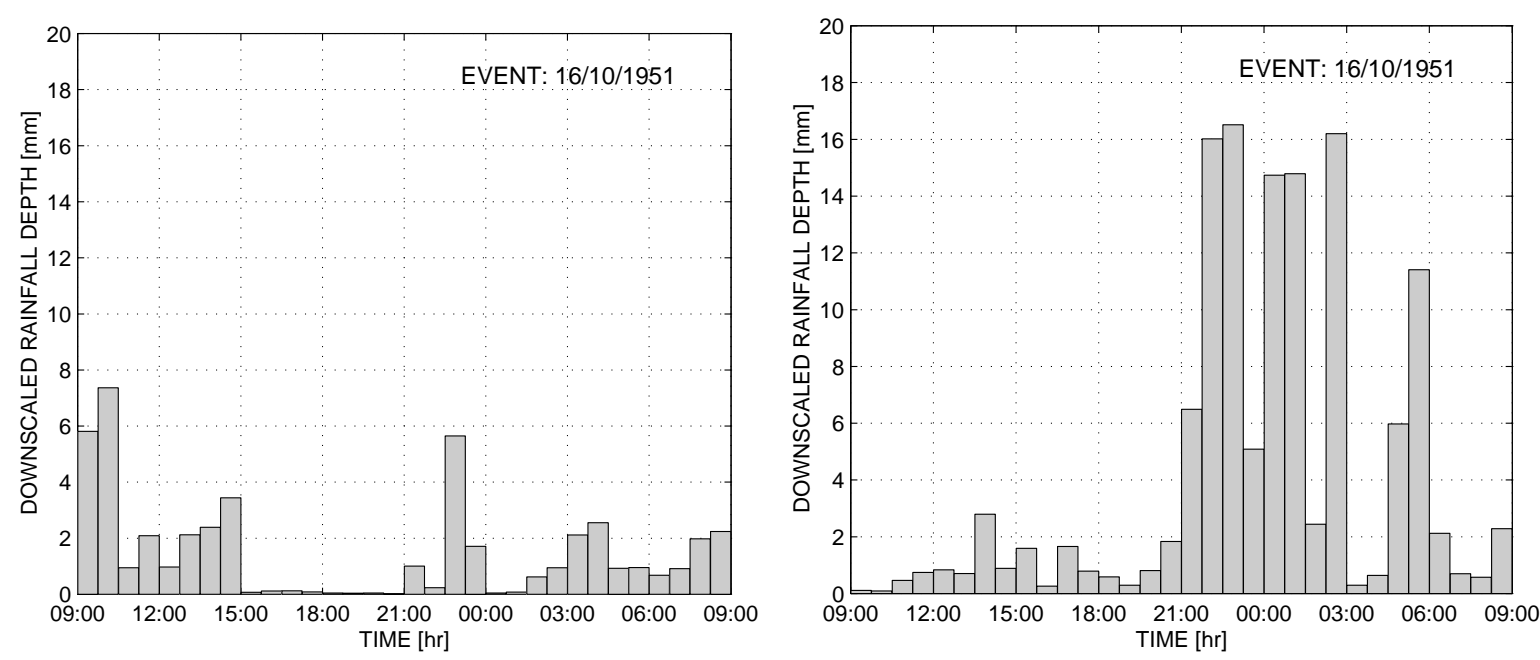

Fig. 2. Two possible scenarios of the same large scale event: synthetic hyetographs generated at the Araxisi catchment scale.
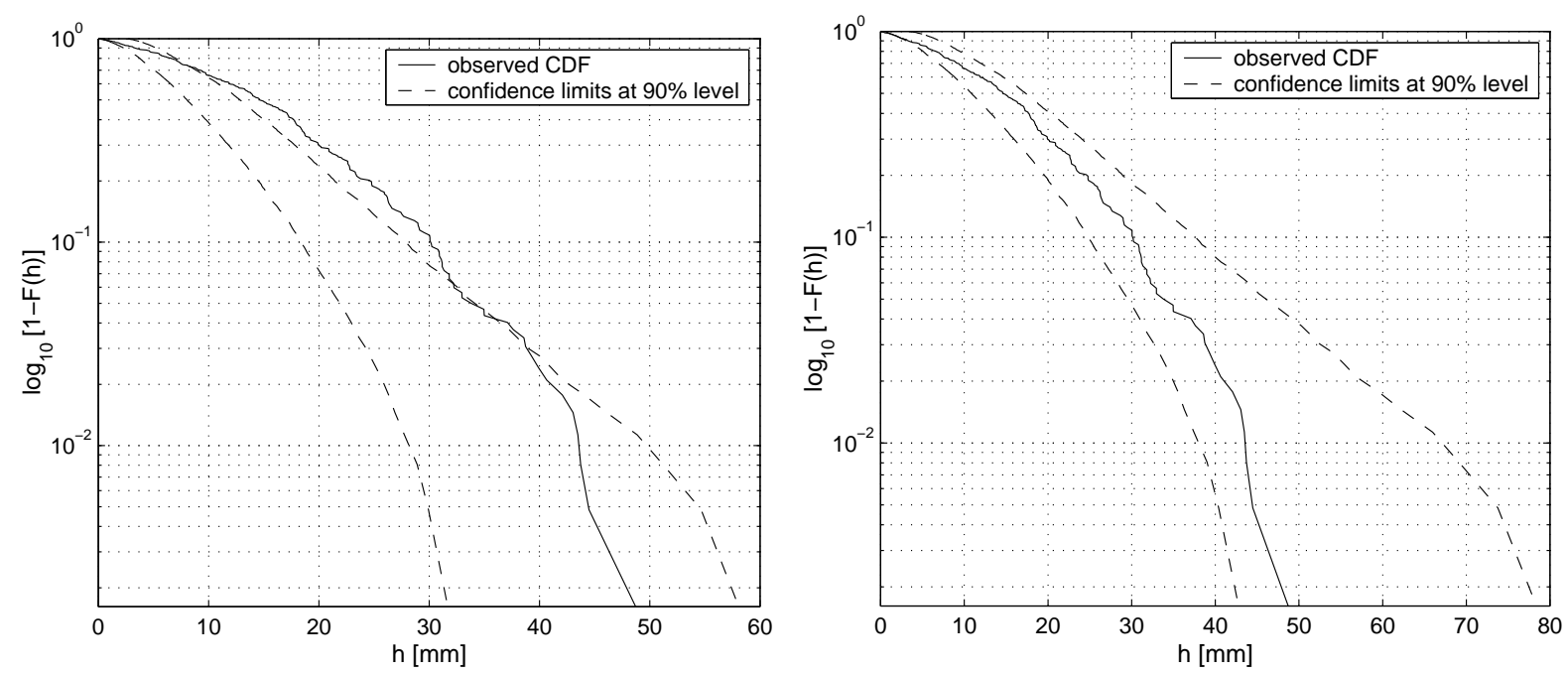

Fig. 3. A comparison between observed and synthetic data is illustrated for events belonging to the third class: the horizontal axis contains daily precipitation values $h$, while the vertical one represents $\log _{10}(1-F(h))$, where $F(h)$ is the CDF. The solid lines represent daily CDF obtained from the control dataset while dashed lines refer to CDFs of the 5\% and $95 \%$ quantiles obtained from generated sequences. In the left plot synthetic data directly come from the STRAIN model, while the modulating function $\bar{\xi}$ is included in data plotted in the right image.

The unit hydrograph theory approximates the catchment response with that of a linear system. The unit hydrograph (UH) is thus the direct runoff hydrograph resulting from a unit depth of excess rainfall at a constant rate for a given (small) time step and occurring uniformly over the catchment. Hydrographs originated from a whatever event are obtained through the convolution of the excess rainfall hyetograph with the unit hydrograph. In the present work three well known lumped UH models were calibrated on the Araxisi catchment: SCS, Clark, Snyder (Chow et al., 1988).

\subsection{R-R model - application}

In Sect. 2.2, for each of the 1303 events a set of 3000 equally probable hyetographs was generated. Here the rainfall-runoff model is applied in order to transform these total rainfall hyetographs into excess rainfall hyetographs (through the SCS-CN module) and then into direct runoff hydrographs at the catchment outlet (by means of the UH model). As a result, 3000 hydrographs for each event were thus obtained applying the SCS, Clark and snyder UH respectively.

The $\mathrm{CN}$ value was determined by subdividing the Araxisi catchment in small areas with homogeneous land-cover and hydrological soil group, and then making a weighted average of the $\mathrm{CN}$ values obtained for each area by standard SCS tables. The final $\mathrm{CN}$ values to be adopted for each event were then corrected, according to the standard SCS-CN method, on the basis of the 5-days antecedent precipitation that is assumed to be representative of the antecedent moisture condition of the catchment. 

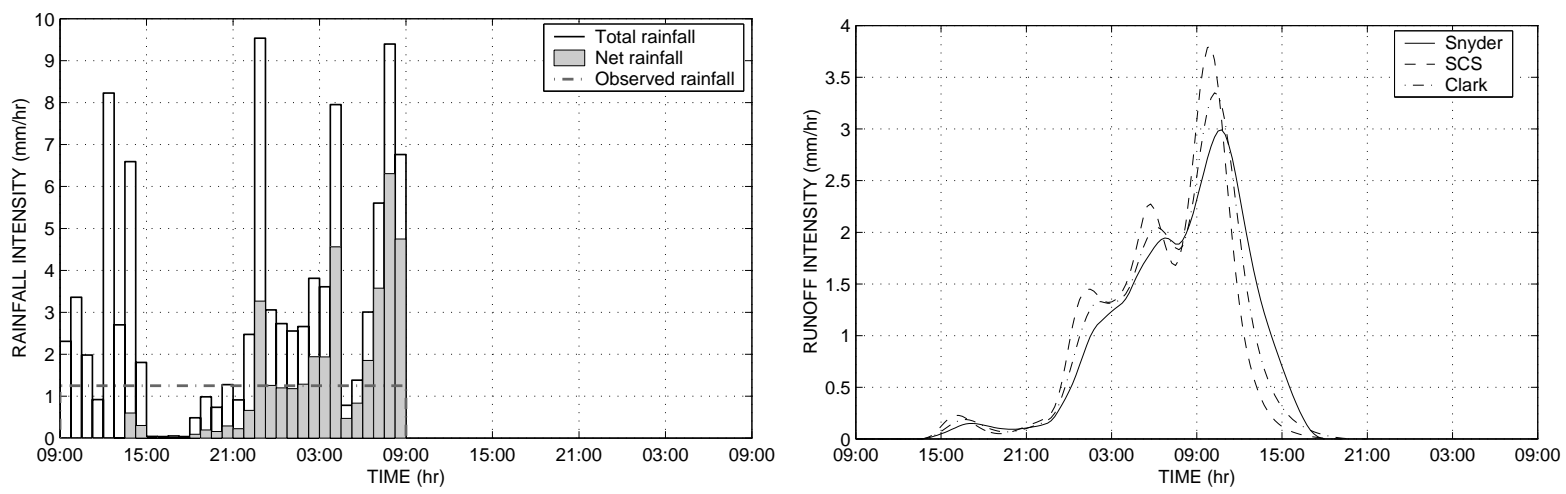

Fig. 4. Left. SCS Curve Number method application: separation of excess rainfall from total rainfall of a downscaled synthetic hyetograph obtained for one of the selected events in the Araxisi catchment (event date: 16/02/1963). Right. Snyder's, Clark's and SCS UH: convolution of the above obtained excess rainfall hyetograph.

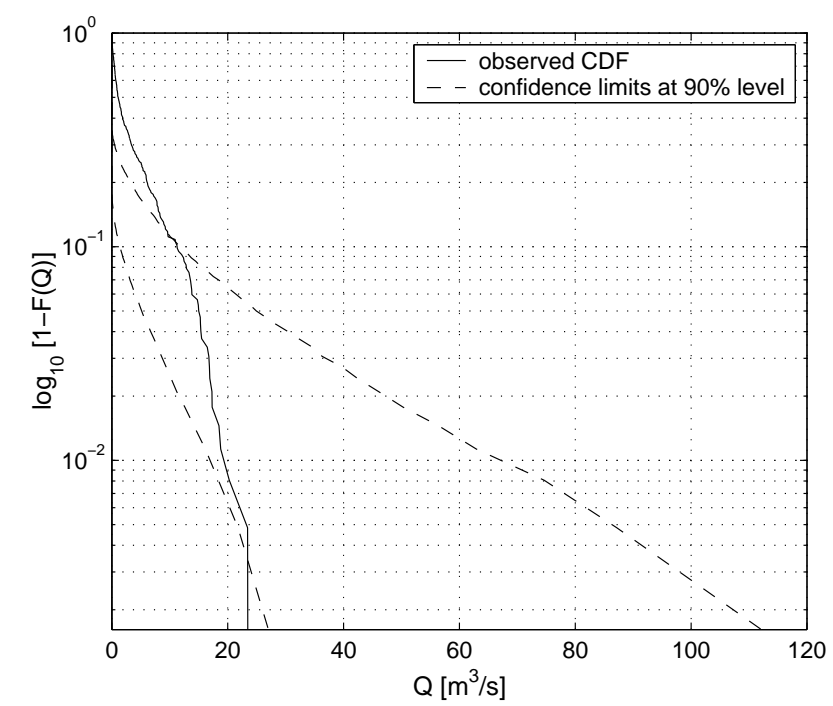

Fig. 5. Empirical CDF (solid line) of flow maxima at 3-hour duration at the outlet of the Araxisi catchment (third class of events). Dashed lines indicates confidence intervals obtained from generated hydrographs (by Clark's UH model) for 5\% and 95\% quantiles. The horizontal axis contains direct runoff values $Q$, while the vertical one represents $\log _{10}(1-F(Q))$, where $F(Q)$ is the CDF.

An application of the rainfall-runoff model to one of the events is illustrated in Fig. 4: the excess rainfall derived by applying the SCS-CN method on a total rainfall synthetic hyetograph obtained from the downscaling model is shown on the left, while the respective synthetic hydrographs obtained with the application of the UH models is presented on the right.

\subsection{R-R model - validation and determination of critical points}

In order to validate the rainfall-runoff model described above, we first extracted the direct runoff component from the hydrographs measured at the basin outlet by applying the digital filter proposed by Nathan and McMahon (1990) for each of the 1303 events considered in Sect. 2.2. In such a way a control dataset of direct runoff hydrographs was constructed for the rainfall-runoff model validation.

For each class of events defined in Sect. 2.3 (Table 1), the global performances of the forecasting chain are investigated by statistical comparison between measured and generated runoff. In particular, flow maxima at different durations are determined from the control dataset of direct runoff and compared with the flow maxima extracted from generated hydrographs. Using an approach similar to the one described in Sect. 2.3, data coming from synthetic generations are used to determine $90 \%$ equitailed confidence intervals for the variable to be checked. As an example, the results obtained for 3-hour flow maxima are illustrated in Fig. 5 for the third class and Clark's UH model. Figure clearly shows how CDF of measured flows lies out and far off the $90 \%$ confidence limits. Unsatisfying results were also obtained for the other classes of events and for flow maxima at different durations. Very small differences were found among SCS, Clark and Snyder UH models, because the three unit hydrographs have similar base times and the convolution effect attenuate their differences.

With the aim to detect the problems that lead to the discrepancies between the features of the generated hydrographs and those of the control dataset highlighted in Fig. 5, each phase of the rainfall-runoff step was analysed in more detail and some diagnostic comparisons were built.

The first aspect to be discussed and investigated regards the effect of the SCS-CN Eq. (4), which is clearly a nonlinear transformation with a lower threshold (when $P<I_{a}$ it returns excess rainfall $P_{e}=0$ ). This means that differences in the probability distributions of total rainfall $P$ may be in principle amplified or reduced in the resulting distributions of excess rainfall derived from Eq. (4). The results of the application of Eq. (4) to downscaled rainfall (on the Araxisi grid cell) were thus compared with those obtained applying the same equation to the control set of daily rainfall. Once more, comparisons were performed in terms of CDFs 


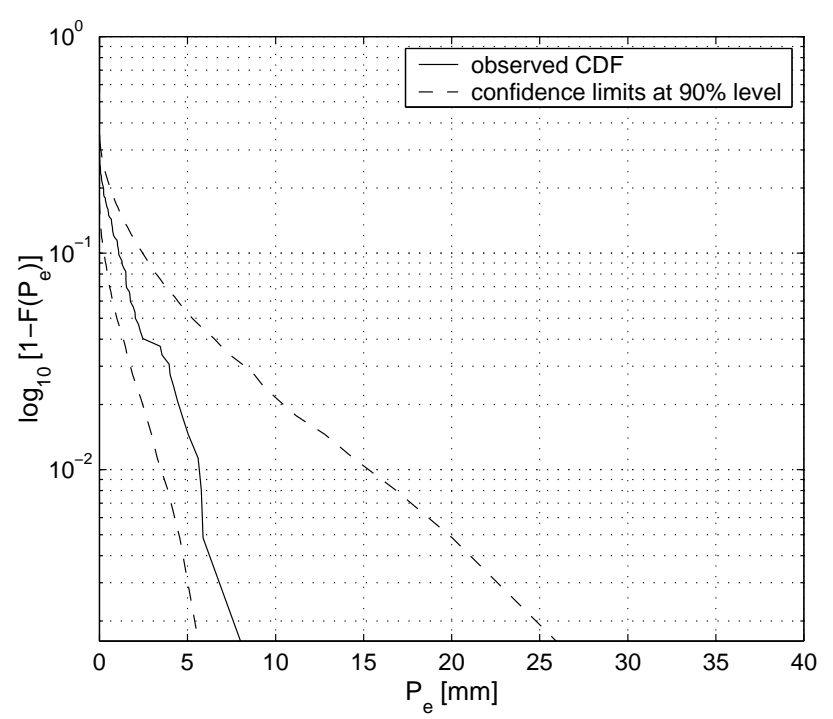

Fig. 6. CDFs of daily excess rainfall $P_{e}$ computed by Eq. (4): results from the control dataset (solid line) are compared with the 90\% confidence intervals obtained from downscaled rainfall on the Araxisi grid cell.

within the classes already introduced in Sect. 2.3 (Table 1). CDFs of excess rainfall obtained from the control dataset resulted always within the confidence limits derived by downscaled data, meaning that the behaviour of SCS-CN equation is very similar for both the observed and the downscaled rainfall. This positive result is mainly due to the ability of the downscaling procedure in reproducing the same variability of observed rainfall at the catchment scale. As an example, Fig. 6 shows the comparison for the third class of the events recorded on the Araxisi catchment.

A second kind of investigations was aimed at comparing the synthetic excess rainfall at daily scale (obtained through the application of Eq. (4) to downscaled rainfall) with the daily discharge extracted from the corresponding direct runoff hydrographs in the control dataset. This comparison, which is illustrated in Fig. 7 for the third class of events, shows that CDFs derived from the control dataset are not included in the confidence limits obtained through generated data. In order to better understand the aim of this comparison and to correctly interpret these results, some consideration are needed. First of all, since comparisons are performed at the daily scale, while the catchment response time is about three hours, the differences between daily excess rainfall depths and the corresponding daily discharge depths obtained by the UH model should be very small. Thus, the comparison of different kinds of variables (excess rainfall and direct runoff) is in this sense allowed and we expect that it does not introduce significant sources of error. Moreover, considering synthetic excess rainfall rather than synthetic direct runoff produced by the rainfall-runoff model allows to exclude potential problems that may arise from the application of the UH model. Thus, problems of rainfall-runoff model have to be searched in the SCS module rather than in the UH one.

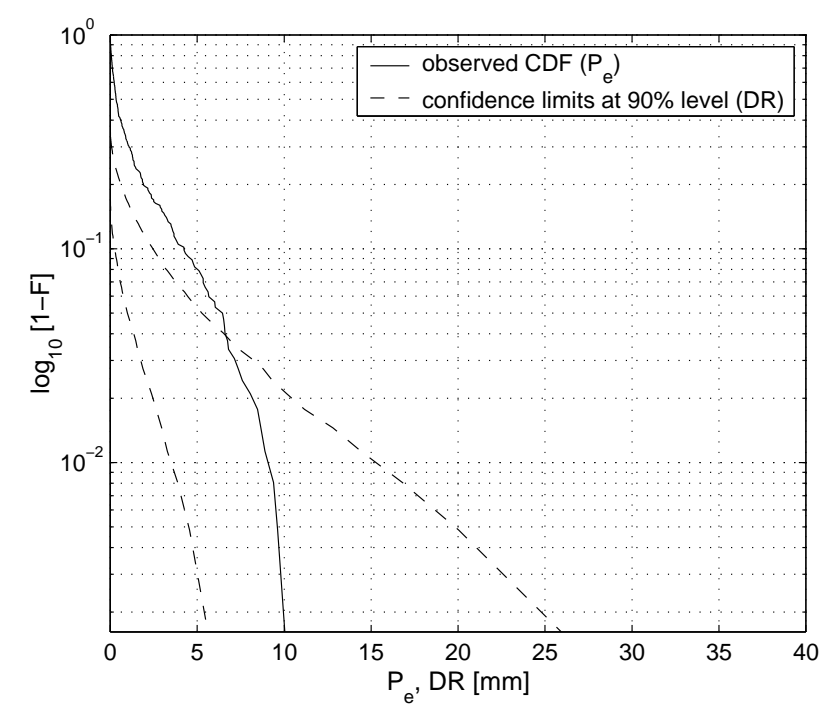

Fig. 7. A comparison between CDFs obtained for direct runoff component $(D R)$ and generated daily excess rainfall $\left(P_{e}\right)$ on the Araxisi catchment is presented. The horizontal axis contains direct runoff $(D R)$ and excess rainfall values $\left(P_{e}\right)$, while the vertical one represents $\log _{10}(1-F)$, where $F$ is the CDF. The solid line represents daily CDF obtained for direct runoff from the control dataset, while dashed lines refer to CDFs obtained for daily excess rainfall from downscaled precipitation for $5 \%$ and $95 \%$ quantiles. The plot refers to the third class of events.

A confirmation of this conclusion comes also from another, and last, kind of comparisons that is based on the scatterplots of the control set of direct runoff discharge at the daily scale against the excess rainfall obtained by the SCSCN Eq. (4) when using the control daily rainfall. We should expect that all the points lie along the bisector line of the scatterplot, but results obtained for the six classes of events clearly show a great dispersion. Figure 8 shows an example for the third class where it is apparent the distance of the points from the bisector line.

We discuss some further considerations about the outcome of this analysis. First, this last comparison is only based on observed data, although they are manipulated and transformed. Thus, results presented in the scatterplot illustrated in Fig. 8 cannot be affected by any kind of errors introduced in the meteo-hydrological forecasting chain by the NWP or the downscaling step. Moreover, apart from the measurement errors of rainfall and streamflow, the only causes of errors are to be ascribed to the direct runoff separation method or to the SCS-CN method. Regarding the streamflow separation method, beside the digital filter by Nathan and McMahon (1990) another attempt was made implementing also traditional separation technique, but despite some slight differences, the scatterplots were as much unsatisfying. Thus we exclude that the main cause of discrepancy could be related to the separation technique, and again we point out the SCS-CN method as the main source of errors.

Finally, in order to improve the performances of the SCS model, a lot of trials for a better calibration of its parame- 


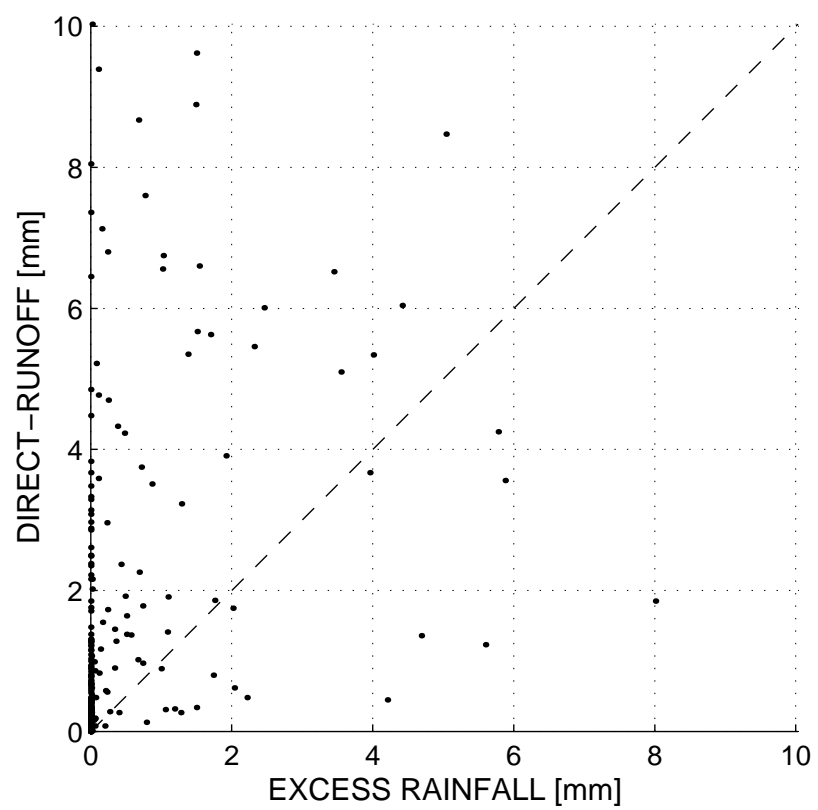

Fig. 8. Scatterplot of observed daily excess rainfall obtained with SCS-CN method against the corresponding direct runoff component obtained with the application of the digital filter on the recorded hydrographs. The comparison is illustrated for the third class of events recorded on the Araxisi catchment.

ters were performed, using data of control dataset. The $\mathrm{CN}$ parameter and also the rainfall thresholds for the three AMC were recalibrated by a least square method on Eq. (4), assuming the couples $\left(P, P_{e}\right)$ to be respectively the rainfall observations and the direct runoff components of the same events. Nevertheless, even applying the rainfall-runoff model with SCS recalibrated parameters, we were not able to significantly improve the unsatisfying results presented above.

\section{Post-processing model (step 4)}

The purpose of a forecasting chain is to assess the hydrological risk caused by predicted heavy rainfall events. In order to face this issue, we propose a simple technique providing a probabilistic framework useful for decision support.

From the set of synthetic hydrographs, generated in the previous step of the forecasting chain, the flow-durationfrequency (FDF) curves are derived for different exceedence probability levels. As an example, results obtained for one of the selected events on the Araxisi catchment using the Clark UH are illustrated in Fig. 9. Once critical streamflow thresholds have been identified for the catchment, the curves FDF provide the probability that these thresholds may be exceeded, and therefore are very useful in supporting decision to issue alerts on the basis of hydrological risk assessment.

Moreover, the FDF curves allow to derive synthetic hydrographs to be used as input in overland flow models in order to simulate downstream overflowing areas. Once a probability level is chosen, a synthetic hydrograph statistically rep-

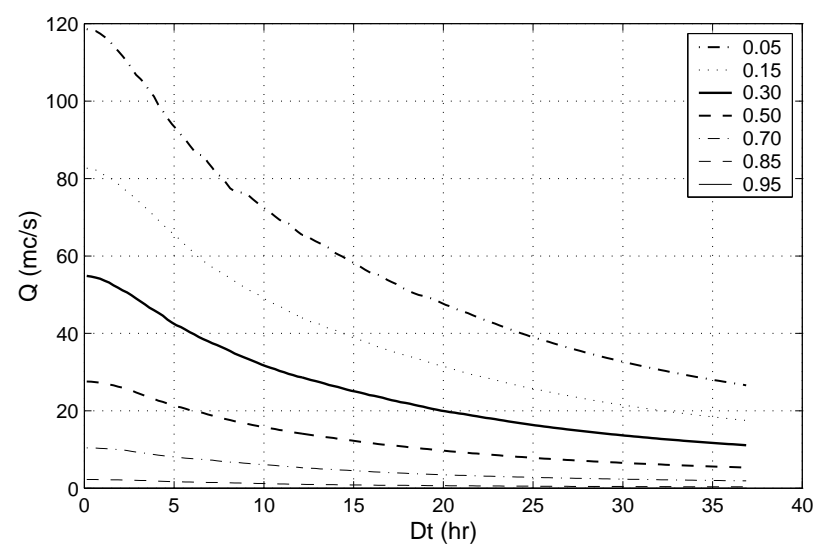

Fig. 9. Flow-duration-frequency curves for different exceedence probability levels are shown. These curves are derived from the set of synthetic hydrographs generated by the forecasting chain (using the Clark UH) for the event of 16/02/1963 on the Araxisi catchment.

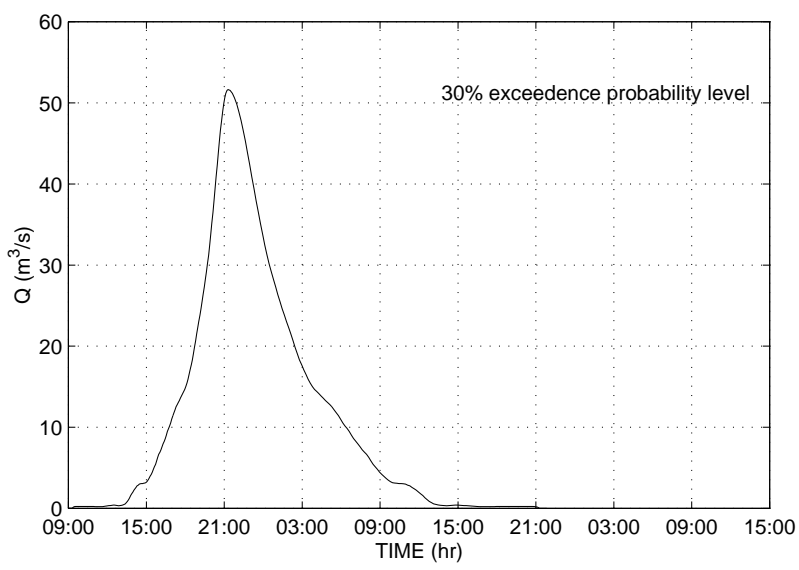

Fig. 10. Outcome of the forecasting chain: a 30\% exceedence probability hydrograph on the Araxisi outlet is obtained subdividing discharges derived from the flow-duration-frequency curve (Fig. 9) accordingly the following proportions: $1 / 3$ on the left-hand side of the peak and $2 / 3$ on the right-hand side.

resentative for predicted discharges at any duration can be obtained. After deriving volumes corresponding to different durations from FDF curves, the synthetic hydrograph can be drawn starting from the flow around the peak, which corresponds to the discharge extracted for the shortest duration, and then subdividing the increments of discharge derived for larger durations between the left and right-hand side of the peak. As an example, Fig. 10 shows the synthetic hydrograph at $30 \%$ exceedence probability level obtained for one of the selected events.

\section{Final remarks}

The proposed procedure can be operatively applied in order to predict and mitigate the adverse effects of meteorological hazards occurring over small watersheds. Inherent in each 
part of a forecasting chain there is a degree of uncertainty. When the modules of the procedure are aggregated, these uncertainties are compounded. Thus it is fundamental to understand the approximations of each step of the procedure and to evaluate any source of error.

Regarding the NWP model, it would be useful to know the probability distributions of errors of rainfall predictions associated at different time-scale resolutions. Actually, identifying the best trade-off between reliability and resolution of forecasted rainfall fields would help in choosing at which space-time scales start the downscaling process. As for the downscaling step, the STRAIN model has, in the proposed configuration, only one free parameter $c$, which can be expressed in terms of a the large scale rainfall intensity $I$, a variable readily available from the previous step. A modulating function $\bar{\xi}$ can be easily introduced in the STRAIN fields to account for spatial rainfall heterogeneity induced by orography. A re-calibration of $c$ and $\bar{\xi}$ may be needed depending on the region and on the large scale of the process.

With respect to the rainfall-runoff model step, several problems were encountered applying the SCS method for excess rainfall separation. Actually this procedure was initially proposed for design flow computation, coupling rainfall depth and discharge amounts with the same return time, but not necessary belonging to the same event. The application of the SCS model to continuous or event based simulations, a common practice in several public domain models, may fail in reproducing excess rainfall as described in the review book by Mishra and Singh (2003) and references therein. This separation technique was identified as the main critical point of the forecasting chain.

In order to give a probabilistic response, the postprocessing module is here proposed. The approach is based on the construction of flow-duration-frequency curves, which allows to assess the risk that some critical streamflow thresholds may be exceeded. Furthermore, the same curves allow the derivation of synthetic hydrographs to be used as input in overland flow models in order to simulate downstream overflowing areas. Both these approaches provide a probabilistic framework helping decision support in managing intense hydrological events occurring over small watersheds.

Acknowledgements. We are grateful to Sardinian Hydrological Survey for making data of the high temporal resolution rain gage network available. The research was supported by the Italian Ministry of Education, University and Research (MIUR) - Programma Operativo Nazionale - Misura 1.3, Prot. 13018/2001.

Edited by: V. Kotroni and K. Lagouvardos Reviewed by: A. Koussis and D. Koutsoyannis

\section{References}

Badas, M. G., Deidda, R., and Piga, E.: Orographic influences in rainfall downscaling, Adv. Geosci., 2, 285-292, 2005, http://www.adv-geosci.net/2/285/2005/.

Badas, M. G., Deidda, R., and Piga, E.: Modulation of homogeneous space-time rainfall cascades to account for orographic influences, Nat. Hazards Earth Syst. Sci., 6, 427437, 2006, http://www.nat-hazards-earth-syst-sci.net/6/427/2006/.

Chow, V. T., Maidment, D. R., and Mays, L. W.: Applied Hydrology, Mc Graw-Hill, New York, 1988.

Deidda, R.: Rainfall downscaling in a space-time multifractal framework, Water Resour. Res., 36, 1779-1794, 2000.

Deidda, R., Benzi, R., and Siccardi, F.: Multifractal modeling of anomalous scaling laws in rainfall, Water Resour. Res., 35, 1853-1867, 1999.

Deidda, R., Badas, M. G., and Piga, E.: Space-time scaling in highintensity Tropical Ocean Global Atmosphere Coupled OceanAtmosphere Response Experiment TOGA-COARE storms, Water Resour. Res., 40, W02 506, doi:10.1029/2003WR002 574, 2004.

Deidda, R., Badas, M. G., and Piga, E.: Space-time Multifractality of Remotely Sensed Rainfall Fields, J. Hydrol., 322, 2-13, doi:10.1016/j.jhydrol.2005.02.036, 2006.

Ferraris, L., Rudari, R., and Siccardi, F.: The uncertainty in the prediction of flash floods in the northern mediterranean environment, J. Hydrometeo., 3, 714-727, 2002.

Gupta, V. K. and Waymire, E. C.: A statistical analysis of mesoscale rainfall as a random cascade., J. Appl. Meteor., 32, 251-267, 1993.

Lovejoy, S. and Mandelbrot, B. B.: Fractal properties of rain and a fractal model., Tellus, 37A, 209, 1985.

Mishra, S. and Singh, V.: Soil Conservation Service Curve Number (SCS-CN) Methodology, Kluwer Academic, 2003.

Nathan, R. and McMahon, T. A.: Evaluation of Automated Techniques for Baseflow and Recession Analysis, Water Resour. Res. 26, 1465-1473, 1990.

Over, T. M. and Gupta, V. K.: A space-time theory of rainfall using random cascades, J. Geophys. Res., 101(D21), 26319-26331, 1996.

Perica, S. and Foufoula-Georgiou, E.: Model for multiscale disaggregation of spatial rainfall based on coupling meteorological and scaling descriptions, J. Geophys. Res., 101, 26347-26361, 1996.

Schertzer, D. and Lovejoy, S.: Physical modeling and analysis of rain and clouds by anysotropic scaling of multiplicative processes, J. Geophys. Res., 92(D8), 9693-9714, 1987.

SCS: National Engineering Handbook - Hydrology, chap. Sect. 4, USDA Soil Conservation Service, Washington, D.C., 1972.

Siccardi, F., Boni, G., Ferraris, L., and Rudari, R.: A hydrometeorological approach for probabilistic flood forecast, J. Geophys. Res., 110, D05 101, doi:10.1029/2004JD005 314, 2005.

Simmons, A. and Hollingsworth, A.: Some aspects of the improvement in skill of numerical weather prediction, Quarterly Journal of the Royal Meteorological Society, 128, 647-678, 2002. 